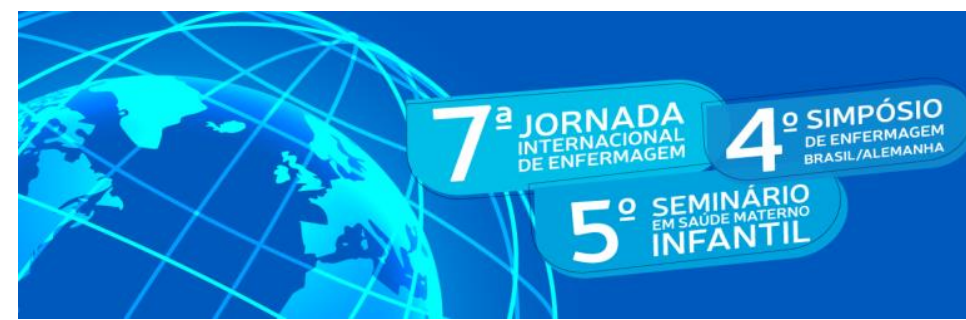

PRÁTICA AVANÇADA EM SAÚDE:

05 a 07.05 de 2021

ISSN: 2318-0234

DOI: http://doi.org/10.48195/jie2021-060

\title{
ANÁLISE DAS TECNOLOGIAS DE EDUCAÇÃO APLICADA NA SAÚDE
}

\section{Yone Gonçalves dos Santos'; Vinicius Lino de Souza Neto²; Renata Santos Solheiro; Sheron Brandão da Silva ${ }^{4}$; Layana Santos Silva ${ }^{5}$; Anderson Varjão De Oliveira ${ }^{6}$}

\begin{abstract}
RESUMO
Objetivo: Analisar conteúdo científico frente as tecnologias de educação aplicada na saúde. Método: Trata-se de um estudo de revisão integrativa da literatura que é composto pelas seguintes etapas: Localização e seleção dos estudos; avaliação crítica dos estudos; coleta de dados; análise e apresentação dos dados; e interpretação dos resultados. Nele foi utilizada as publicações científicas disponibilizadas nas bases de dados BVS, PubMed e Scielo. Resultados: Após análise, a amostra final foi de 19 artigos e observou-se uma grande importância das tecnologias educativas para promover saúde, estando entre elas: jogos, vídeos, aplicativos, cursos, websites, dispositivos móveis e cartilhas. Conclusão: Por fim, foi evidenciado que tais tecnologias são essenciais para educação em saúde, uma vez que, educar não é uma tarefa fácil e requer meios mais didáticos para sua elaboração.
\end{abstract}

Palavras-chave: Tecnologias de educação em saúde; Educação em saúde; Tecnologias educativas no mundo.

\begin{abstract}
Objective: Analyze scientific content in the face of applied education technologies in health. Method: This is a integrative literature review study that consists of the following steps: Location and selection of studies; critical evaluation of studies; data collect; analysis and presentation of data; and interpretation of results. It used the scientific publications available in the VHL, PubMed and Scielo databases. Results: After analysis, the final sample was 19 articles and there was a great importance of educational technologies to promote health, including: games, videos, applications, courses, websites, mobile devices and booklets. Conclusion: Finally, it was evidenced that such technologies are essential for health education, since educating is not an easy task and requires more didactic means for its elaboration.
\end{abstract}

\footnotetext{
${ }^{1}$ Estudante do Curso Bacharelado em Enfermagem. Universidade Nove de julho. E-mail: yonegoncalves16@gmail.com

${ }^{2}$ Orientador. Doutorando em Enfermagem pela Universidade Federal de São Paulo - UNIFESP. Universidade Nove de Julho. E-mail: vinolino@hotmail.com

${ }^{3}$ Estudante do Curso Bacharelado em Enfermagem. Universidade Nove de julho. E-mail: solheirorenata@gmail.com

${ }^{4}$ Estudante do Curso Bacharelado em Enfermagem. Universidade Nove de julho. E-mail: sheron.brandao.sb@gmail.com

${ }^{5}$ Estudante do Curso Bacharelado em Enfermagem. Universidade Nove de julho. E-mail: layanasasilva@gmail.com

${ }^{6}$ Estudante do Curso Bacharelado em Enfermagem. Universidade Nove de julho. E-mail: andersondiscord@outlook.com
} 


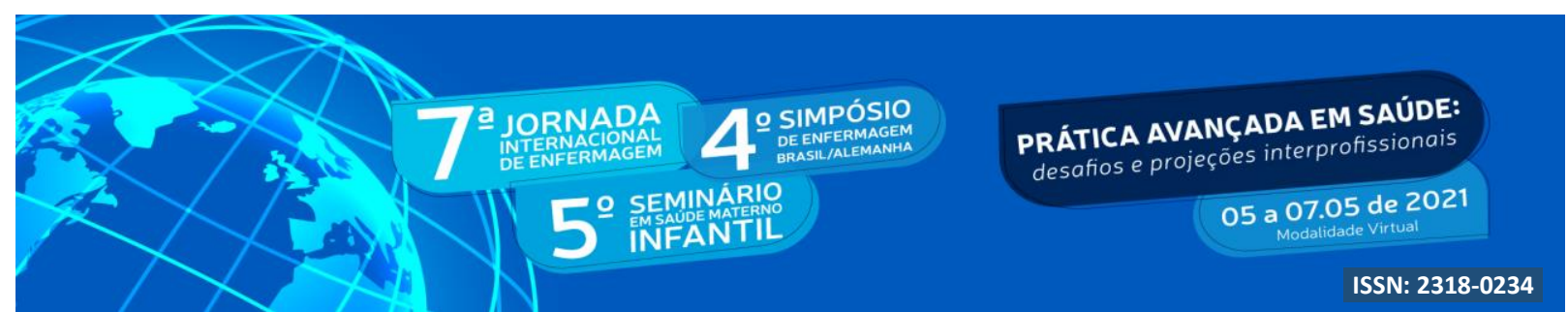

Key Words: Health education technologies; Health education; Educational technologies in the world.

\section{INTRODUÇÃ̃O}

A expressão "tecnologia educativa" refere-se ao uso de recursos tecnológicos como instrumento para o ensino e aprendizagem, seu objetivo é apoiar os métodos já existentes e oportunizar um melhor acesso à informação (PORTAL, et al., 2020).

Nesse contexto, as tecnologias educativas são meios inovadores capazes de modificar indivíduos e ambientes, proporcionando mudanças na realidade e invertendo situações problemas. A educação em saúde é de suma importância para a vida de toda uma comunidade, sendo ela a base e o suporte para todo o seu processo de construção (BATISTA NETO, et al., 2020).

As tecnologias de educação em saúde são apresentadas como um aglomerado de ações e estudos voltados para a prevenção, promoção e recuperação da saúde. São utilizados panfletos, cartilhas, vídeos, campanhas, cursos, rodas de conversa, cartazes, multimídias, diálogos e equipamentos que contribuem significativamente, para o desenvolvimento comunitário (BATISTA NETO, et al., 2020).

Estudos acerca da aplicabilidade dessas tecnologias no mundo vem ganhando espaço, uma vez que esse processo de educação em saúde é substancial para o fortalecimento da interação entre o profissional e a comunidade. De fato, com o crescimento das tecnologias educacionais em saúde é evidenciado uma melhoria quanto aos cuidados em saúde (SILVA, et al., 2020).

Entretanto, cabe ressaltar, que é imprescindível a ampliação de investimentos que abrangem essa temática, posto que, determinadas ações e propostas influenciam de maneira positiva na promoção da saúde.

\section{OBJETIVO}

Analisar conteúdo científico frente as tecnologias de educação aplicada na saúde. 


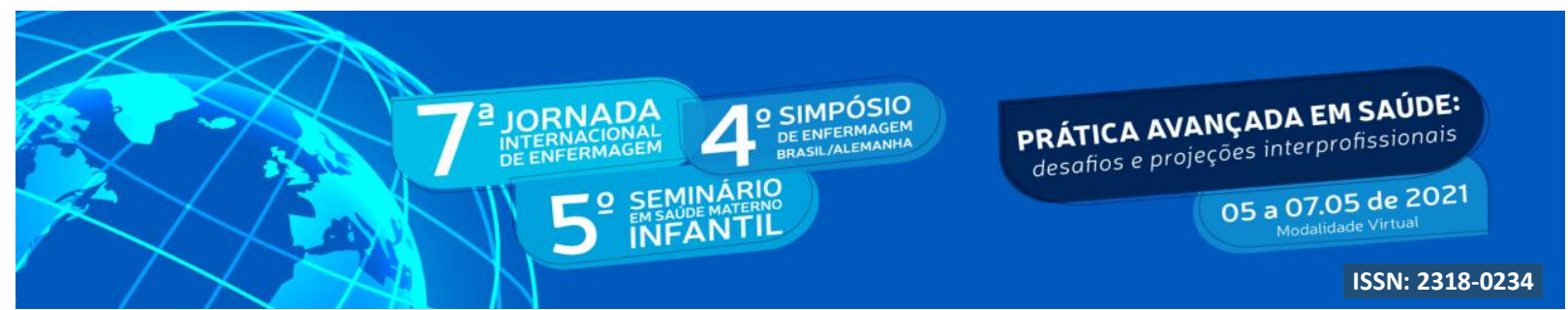

\section{METODOLOGIA}

Trata-se de um estudo de revisão integrativa da literatura que é composto pelas seguintes etapas: Localização e seleção dos estudos; avaliação crítica dos estudos; coleta de dados; análise e apresentação dos dados; e interpretação dos resultados.

Assim a pergunta que norteou a pesquisa foi: Quais as tecnologias educacionais mais postas em práticas no mundo? A coleta de dados ocorreu nos meses de fevereiro e março de 2021 por meio do acesso online as bases de dados Pubmed, Scielo e Cinahl e foram utilizados os seguintes descritores: "Tecnologias de educação em saúde", "Educação em saúde" e “Tecnologias educativas". Para selecionar os artigos na revisão, foram adotados os seguintes critérios: estudos com o foco em apresentar tecnologias educativas, independentemente do idioma, do tipo de tecnologia e do setor de aplicação. Os critérios de exclusão foram: pesquisa que não evidenciasse tecnologias para educação.

Portanto, por meio da estratégia de busca, foram identificados 56 artigos na Cinahl, 26 na PubMed e 14 na Scielo, totalizando 96 estudos. Ademais, foi feita a seleção usando os critérios já citados, após isso, com o objetivo de filtrar melhor as pesquisas, analisaram os títulos, resumos e a íntegra, deixando apenas 19 artigos que se enquadraram no escopo da pesquisa.

Com o número de artigos coletados e já selecionados, foi utilizado um instrumento para analisar a qualidade dos estudos selecionados. Os autores utilizados nessa pesquisa foram devidamente referenciados, respeitando e identificando as fontes de pesquisa, observando o rigor ético quanto à propriedade intelectual dos textos científicos que foram analisados, no que se refere ao uso do conteúdo e de citação das partes das obras consultadas.

\section{RESULTADOS E DISCUSSÃO}

A amostra final foi de 19 artigos, analisados, primeiramente, por meio da leitura do título e resumo e logo após, resultados e discussões. Evidencia-se que em todos os níveis da assistência, o uso das tecnologias de educação em saúde é primordial. Para isso, é preciso que 


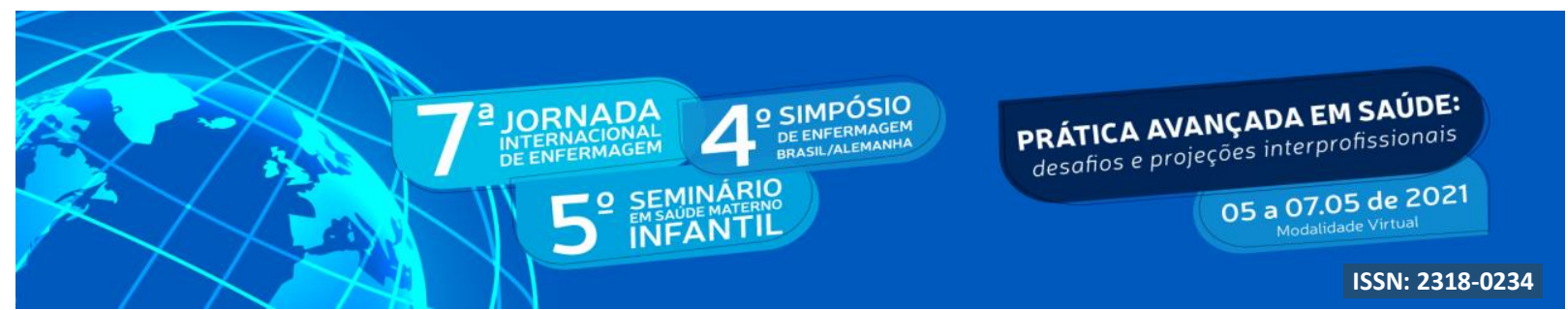

sejam acessíveis e integral para todos. Assim, na figura 1, observa-se sete tipos de tecnologias educativas, como também o seu objetivo e o que ela promove.

Figura 1: Análise das tecnologias educativas.

\begin{tabular}{|c|c|c|}
\hline Jogos & Aprender brincando & $\begin{array}{c}\text { Contribue para a } \\
\text { interação social e } \\
\text { ajuda na } \\
\text { identificação dos } \\
\text { problemas }\end{array}$ \\
\hline Vídeos & $\begin{array}{c}\text { Tornar o } \\
\text { aprendizado mais } \\
\text { criativo }\end{array}$ & $\begin{array}{c}\text { Promove o estímulo } \\
\text { audiovisual e torna a } \\
\text { maneira de } \\
\text { aprender mais } \\
\text { dinâmica e } \\
\text { produtiva }\end{array}$ \\
\hline Aplicativos & $\begin{array}{l}\text { Disseminar } \\
\text { informações }\end{array}$ & $\begin{array}{c}\text { Promove um } \\
\text { aprendizado } \\
\text { eficiente, versátil e } \\
\text { amplo }\end{array}$ \\
\hline Cartilhas & Guiar as orientações & $\begin{array}{l}\text { Auxilia e reforça as } \\
\text { informações para as } \\
\text { tomadas de decisões }\end{array}$ \\
\hline Cursos & $\begin{array}{l}\text { Aperfeiçoar o } \\
\text { conhecimento no } \\
\text { assunto }\end{array}$ & $\begin{array}{c}\text { Possibilidade de } \\
\text { suprir uma carencia } \\
\text { de conhecimento e se } \\
\text { capacitar em alguma } \\
\text { área }\end{array}$ \\
\hline Dispositivos móveis & $\begin{array}{c}\text { Facilitar a dinamica } \\
\text { entre profissional e } \\
\text { usuário }\end{array}$ & $\begin{array}{c}\text { Promove a } \\
\text { comunicação, } \\
\text { facilita na didática e } \\
\text { possibilita uma } \\
\text { apliação dos estudos }\end{array}$ \\
\hline Websites & $\begin{array}{c}\text { Ampliar a busca por } \\
\text { informações }\end{array}$ & $\begin{array}{c}\text { Promove a } \\
\text { pluridisciplinaridad } \\
\text { e, possibilita uma } \\
\text { educação global e } \\
\text { estimula a busca por } \\
\text { conhecimento }\end{array}$ \\
\hline
\end{tabular}




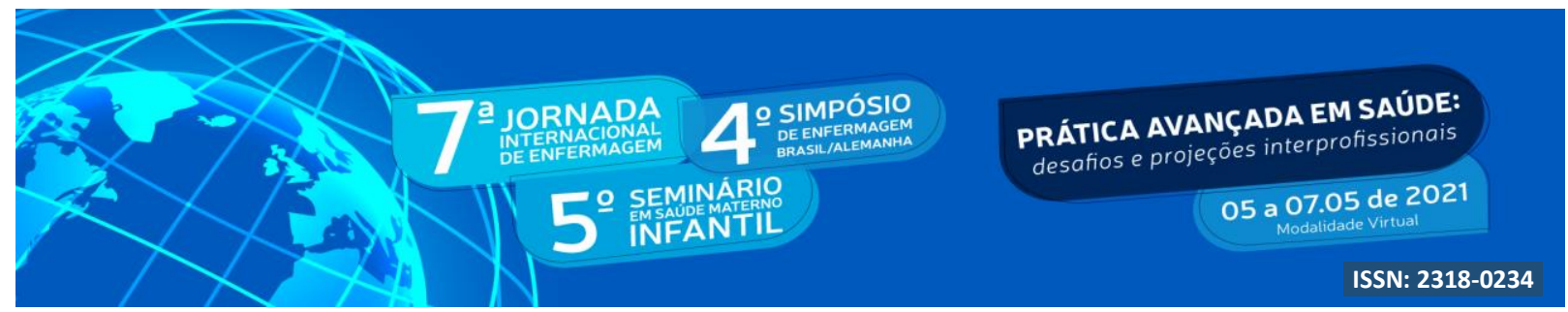

A análise detalhada dos estudos expostos acima apresenta os mais diversos tipos de tecnologias educativas em saúde e as suas inúmeras aplicações. Pesquisas demonstram que a aplicabilidade dessas tecnologias no mundo é essencial para potencializar a disseminação das informações e promover saúde (THOMAS, FONTANA, 2020; GOMES, et al., 2017).

Nesse estudo, a partir da análise detalhada e comparativa dos mais diversos tipos de tecnologias, ficou evidente a necessidade do seu uso no mundo. Entretanto, educar em saúde não é uma tarefa fácil e muitas tecnologias educativas encontram barreiras para serem implementadas (PALÁCIO, TAKENAMI, 2020; SILVA, et al., 2019).

Nesse contexto, evidencia-se que as tecnologias educativas apresentam eficácia no processo de prevenção ou tratamento de determinadas doenças. De fato, essa maneira de educar em saúde se mostrou como algo alternativo, visto que, utilizando métodos menos robótico de ensino torna-se a aprendizagem mais dinâmica e estratégica. Ficou notório também, que em muitos casos quando o cliente toma conhecimento da doença, ocorre uma maior adesão ao tratamento, consequentemente, aumento da qualidade de vida (OLIVEIRA, et al., 2021; BUENO, BUENO, MOREIRA, 2020).

Ademais, é substancial ressaltar que com a utilização de atividades mais lúdicas para o ensino, o profissional estará trabalhando a autonomia daquele sujeito. Dessa maneira, muitos estudos retrataram que com a utilização de jogos, vídeos, cartazes, dinâmicas, bingos, folhetos, etc., ocorre um maior esclarecimento sobre o assunto abordado. Porém, assim como mostrado em um dos estudos, é imprescindível se atentar ao público alvo, uma vez que pode ocorrer inadequação do material ou incapacidade de leitura do próprio leitor (PESSOA, et al., 2019; CYRINO, et al., 2016).

Sob esse viés, através da análise das referidas pesquisas, foi percebido um grande uso de aplicativos para o ensino. O WhatsApp, o Instagram e o Blog se mostraram aliados do processo educativo, pois, reforçaram a comunicação entre profissional e paciente, aumentou a qualidade do cuidado prestado, foi utilizado como meio de divulgação em saúde, além de possibilitar o agendamento de consultas, exames e outros, tornando assim, o processo saúdedoença mais seguro, assertivo e integral (CHAVES, et al., 2018; PAULINO, et al., 2018; AGUIAR, et al., 2018; BERNARDES, et al., 2018; OLIVEIRA, ALENCAR, 2017). 


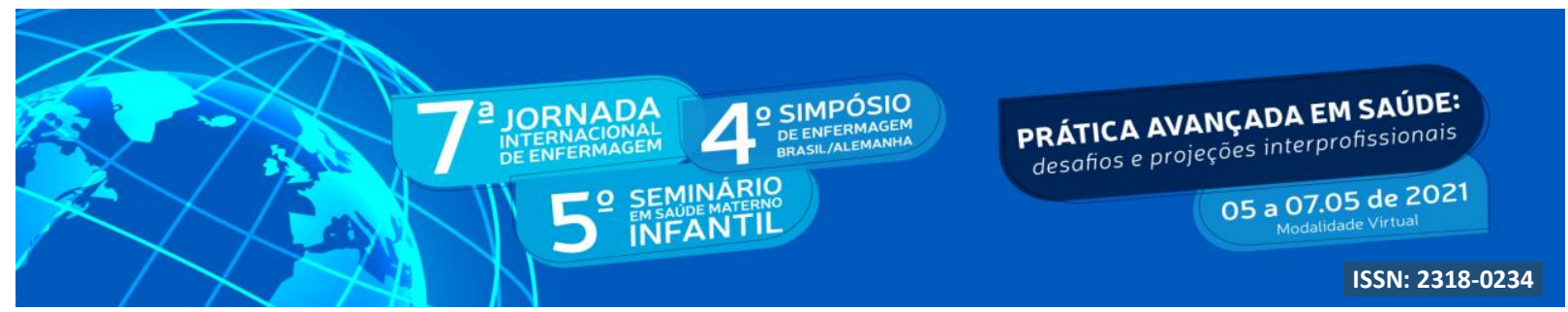

Em meio a utilização dessas ferramentas, a aprendizagem e o desenvolvimento de quem pratica é evidente. Além disso, foi exibido nos estudos que a prática dessas atividades é essencial para fortalecer o vínculo, a confiança e o respeito entre profissional e usuário. À vista disso, de modo a manter e estimular determinadas práticas, os profissionais capacitados devem estar sempre atualizando as formas de ensino, para assim, promover saúde (OLIVEIRA, GONTIJO, MONTEIRO, 2017).

No estudo "Educação e Saúde em crianças com Síndrome de Down” foi retratado o quão benéfico são essas tecnologias para esse público. Nele foi esclarecido que através dos jogos as crianças tiveram um enorme desenvolvimento cognitivo, motor e sensorial, além de promover a interação social com outras crianças do meio. Sendo assim, é preciso rever os investimentos nesta área com o intuito de promover e ampliar esses métodos educativos (CARVALHO, et al., 2017).

Desse modo, olhando para o contexto de pandemia, através da pesquisa "Em tempos de pandemia pela COVID-19: o desafio para a educação em saúde", foi retratada a dificuldade dos profissionais e pesquisadores em saúde para disseminar informações, seja pelos grandes incidentes de Fake News, principalmente nessa última década, ou pelo acúmulo de responsabilidades e tarefas nos profissionais, podendo desenvolver, também., a Síndrome de Burnout (PALÁCIO, TAKENAMI, 2020; SILVA, et al., 2017).

Fica evidente, portanto, que em todos os níveis da assistência, o uso das tecnologias de educação em saúde é primordial. Para isso, é preciso que elas sejam acessíveis e integral para todos. Educação em saúde é algo difícil, que enfrenta inúmeros obstáculos, porém, os benefícios que ela nos proporciona é capaz de superar tais desafios.

\section{CONCLUSÃO}

Nesse contexto, após análise dos estudos expostos foi notado que a aplicação das mais diversas formas de tecnologias educativas no mundo torna o processo de aprendizagem mais prazeroso. Além disso, contribuem significativamente para prevenção e promoção da saúde, assegurando uma assistência integral, segura e assertiva. 


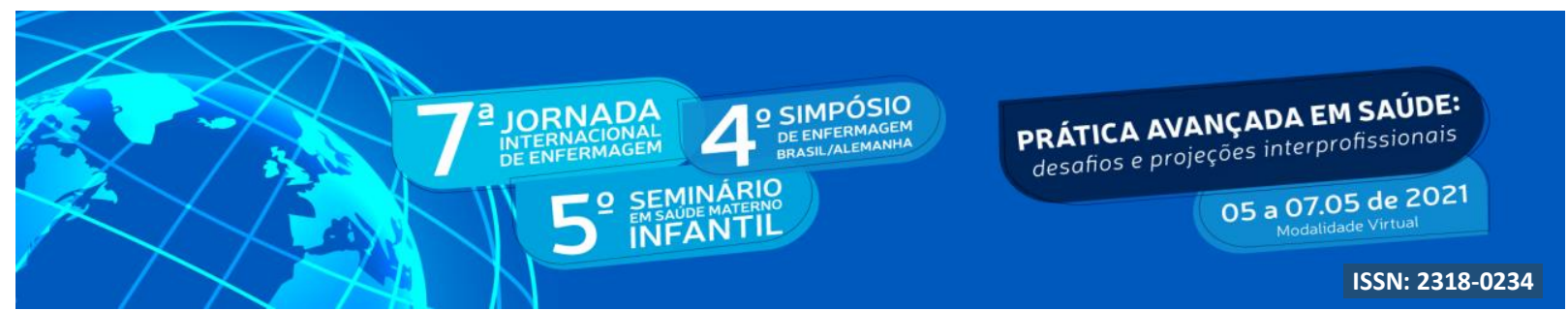

Portanto, a aplicação de jogos, multimídias, vídeos, cartilhas, cursos, aplicativos e campanhas, são exemplos de tecnologias usadas no Brasil e no mundo para a manutenção das diversas formas de cuidado e aplicadas nos diferentes contextos, seja para a prevenção de uma patologia ou, para conscientizar sobre a importância da vacinação, como para o tratamento de um câncer.

\section{REFERÊNCIAS}

AGUIAR, Ana Caroline Leite de et al. Blog como ferramenta educacional: contribuições para o processo interdisciplinar de educação em saúde. Revista Eletrônica de Comunicação, Informação e Inovação em Saúde, v. 12, n. 2, p. 220-31, 29 jun. 2018. Disponível em: https://www.reciis.icict.fiocruz.br/index.php/reciis/article/view/1301/2209 Acessado em: 12 de fevereiro de 2021.

BATISTA NETO, José Benedito dos Santos et al. Construção de tecnologias educativas como forma de educação em saúde para a prevenção da Covid-19: relato de experiência. Revista Eletrônica Acervo Saúde: Electronic Journal Collection Health, Pará, v. 12, n. 9, p. 21782091, 2020.2 Disponível em: https://acervomais.com.br/index.php/saude/article/view/3737/2711 Acessado em 03 de março de 2021.

BERNARDES, Raissy Alves et al. O Instagram como ferramenta para educação em saúde: relato de experiência. I Congresso Norte-Nordeste de Tecnologias em Saúde: 05 a 08 de dezembro, Teresina, v. 1, n. 1, 2018. Disponível em: https://comunicata.ufpi.br/index.php/connts/article/view/7914/4645 Acessado em: 11 de fevereiro de 2021.

BUENO, Maria Bethânia Tomaschewski; BUENO, Mateus Moreira; MOREIRA, Maria Isabel Giusti. Fisioterapia e a educação em saúde. Revista Thema, v. 17, n. 3, p. 675-685, 30 set. 2020. Disponível em: http://periodicos.ifsul.edu.br/index.php/thema/article/view/1594/1563 Acessado em 10 de fevereiro de 2021.

CARVALHO, Marla Dore et al. Ferramentas aplicadas à Educação e Saúde em crianças com Síndrome de Down. III Seminário de Tecnologias Aplicadas em Educação em Saúde: Staes, p. 1-9, 2017. Disponível em: https://www.revistas.uneb.br/index.php/staes/article/view/3828 Acessado em: 20 de fevereiro de 2021.

CHAVES, Arlane Silva Carvalho et al. Uso de aplicativos para dispositivos móveis no processo de educação em saúde: reflexos da contemporaneidade. Revista Humanidades \& Inovação, Palmas-Tocantins, v. 5, n. 6, p. 2358-8322, 2018. Disponível em: 


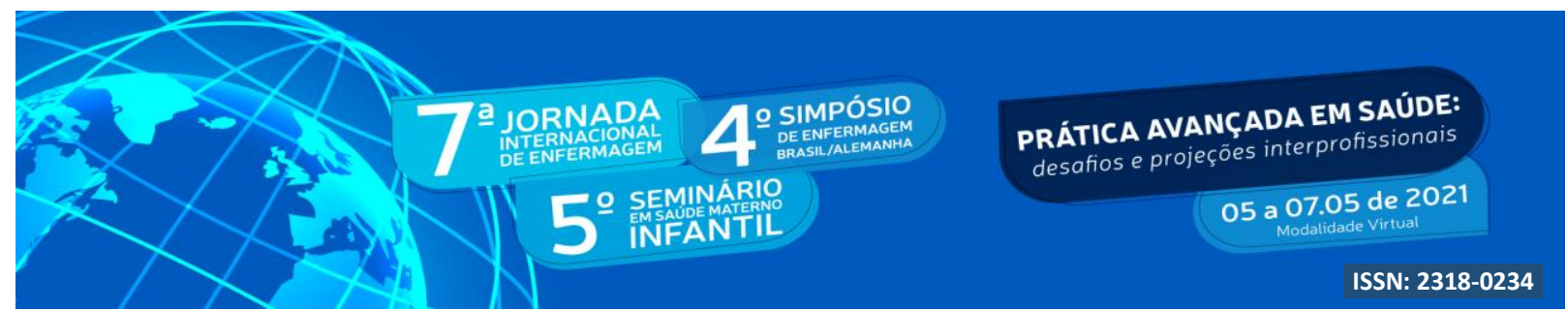

https://revista.unitins.br/index.php/humanidadeseinovacao/article/view/744 Acessado em: 14 de fevereiro de 2021.

CYRINO, Renata Souza et al. Atividades lúdicas como estratégia de educação em saúde com idosos. Revista Ciência em Extensão: Universidade Estadual Paulista, v. 12, n. 3, p. 154163, 2016. Disponível em: https://ojs.unesp.br/index.php/revista_proex/article/view/1324/1260 Acessado em: 10 de fevereiro de 2021.

GOMES, Andréa Tayse de Lima et al. Tecnologias aplicadas à segurança do paciente: uma revisão bibliométrica. Revista de Enfermagem do Centro-Oeste Mineiro, v. 7, p. 1-11, 2017. Disponível em: $\quad$ http://www.seer.ufsj.edu.br/index.php/recom/article/view/1473/1719 Acessado em: 22 de fevereiro de 2021.

OLIVEIRA, Ana Rachel Fonseca de; ALENCAR, Maria Simone de Menezes. O uso de aplicativos de saúde para dispositivos móveis como fontes de informação e educação em saúde. Revista Digital Biblioteconomia e Ciência da Informação, Campinas, SP, v. 15, n. 1, p. 234245, 2017.2 Disponível em: https://periodicos.sbu.unicamp.br/ojs/index.php/rdbci/article/view/8648137/15054 Acessado em: 10 de fevereiro de 2021.

OLIVEIRA, Gabriela et al. Tecnologias voltadas para a hipertensão arterial sistêmica: análise documental da produção de conhecimento no Brasil. Research, Society And Development, v. 10 , n. $1, \quad$ p. 2525-3409, 2021. Disponível em: https://rsdjournal.org/index.php/rsd/article/view/11624/10395 Acessado em: 06 de março de 2021.

OLIVEIRA, Marcela Paula C.; GONTIJO, Daniela Tavares; MONTEIRO, Rosana Juliet Silva. Utilização de uma tecnologia educativa no processo de ensino-aprendizagem de adolescentes sobre saúde sexual e reprodutiva: relatos de experiência. Anais do XXIII Workshop de Informática na Escola, p. 1-9, 27 out. 2017. Disponível em: https://brie.org/pub/index.php/wie/article/view/7229/5027 Acessado em: 04 de março de 2021.

PALÁCIO, Maria Augusta Vasconcelos; TAKENAMI, Iukary. Em tempos de pandemia pela COVID-19: o desafio para a educação em saúde. Vigilância Sanitária em Debate, Paulo Afonso, Ba, Brasil, v. 8, n. 2, p. 10-15, 29 maio 2020. Disponível em: https://visaemdebate.incqs.fiocruz.br/index.php/visaemdebate/article/view/1530/1147

Acessado em: 22 de fevereiro de 2021.

PAULINO, Danilo Borges et al. WhatsApp ${ }^{\circledR}$ como Recurso para a Educação em Saúde: Contextualizando Teoria e Prática em um Novo Cenário de Ensino-Aprendizagem. Rev. bras. educ. med., Brasília, v. 42, n. 1, p. 171-180, jan. 2018. Disponível em: https://www.scielo.br/scielo.php?pid=S0100-55022018000100171\&script=sci_arttext

Acessado em 13 de fevereiro de 2021. 


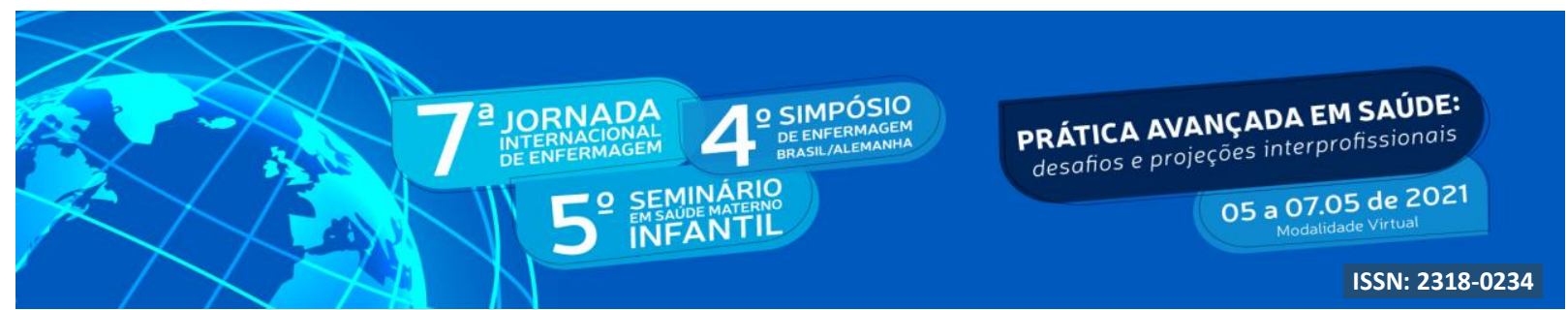

PESSOA, Natália Ramos Costa et al. Tecnologias Educacionais Direcionadas para Pacientes Renais Crônicos na Promoção do Autocuidado. Revista Online de Pesquisa, v. 11, n. 3, p. 756-762, 2019.2 Disponível em: http://www.seer.unirio.br/index.php/cuidadofundamental/article/view/6817/pdf_1 Acessado em: 10 de fevereiro de 2021.

PORTAL, Lorena de Castro et al. Educar para empoderar: o uso de tecnologias educativas para o controle e prevenção de infecção hospitalar. Brazilian Journal Of Development, Curitiba, v. 6, n. 7, p. 50658-50673, jul. 2020. Disponível em: https://www.brazilianjournals.com/index.php/BRJD/article/view/13822/11565 Acessado em: 03 de março de 2021.

SILVA, Camila Tahis dos Santos et al. Tecnologias voltadas para educação em saúde: o que temos para a saúde dos idosos? II Staes. Seminário de Tecnologias Aplicados A Educação e Saúde, Bahia, p. 1-8, 2020. Disponível em: https://www.revistas.uneb.br/index.php/staes/article/view/1615 . Acessado em 03 de março de 2021.

SILVA, Cleyton Cézar Souto et al. Burnout e tecnologias em saúde no contexto da enfermagem na Atenção Primária à Saúde. Esc. Anna Nery, Rio de Janeiro, v. 21, n. 2, e20170031, 2017. Disponível em: $\quad$ https://www.scielo.br/scielo.php?pid=S1414-

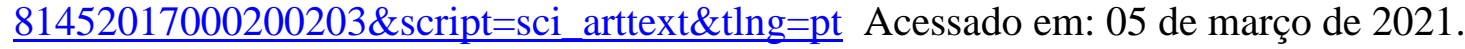

SILVA, Naélia Vidal de Negreiros da, et al. Tecnologias em saúde e suas contribuições para a promoção do aleitamento materno: revisão integrativa da literatura: Ciência \& Saúde Coletiva, v. 24, n. 2, p- 589-602, fev. 2019. Disponível em: https://www.scielosp.org/article/csc/2019.v24n2/589-602/pt/ Acessado em: 20 de fevereiro de 2021.

THOMAS, Larissa Scheeren; FONTANA, Rosane Teresinha. Uso das Tecnologias de Informação e Comunicação como meio educacional na saúde: revisão integrativa. Research, Society And Development, v. 9, n. 10, p. 2525-3409, 29 out. 2020. Disponível em: https://rsdjournal.org/index.php/rsd/article/view/9321/8440 Acessado em: 10 de fevereiro de 2021 